\title{
Generic Bound Coherence under Strictly Incoherent Operations
}

\author{
Ludovico Lami, ${ }^{*}$ Bartosz Regula, ${ }^{\dagger}$ and Gerardo Adesso ${ }^{\ddagger}$ \\ School of Mathematical Sciences and Centre for the Mathematics and Theoretical Physics of Quantum Non-Equilibrium Systems, \\ University of Nottingham, University Park, Nottingham NG7 2RD, United Kingdom
}

(Received 23 September 2018; revised manuscript received 25 January 2019; published 19 April 2019)

\begin{abstract}
We compute analytically the maximal rates of distillation of quantum coherence under strictly incoherent operations (SIO) and physically incoherent operations (PIO), showing that they coincide for all states, and providing a complete description of the phenomenon of bound coherence. In particular, we establish a simple, analytically computable necessary and sufficient criterion for the asymptotic distillability under SIO and PIO. We use this result to show that almost every quantum state is undistillable_-only pure states as well as states whose density matrix contains a rank-one submatrix allow for coherence distillation under SIO or PIO, while every other quantum state exhibits bound coherence. This demonstrates the fundamental operational limitations of SIO and PIO in the resource theory of quantum coherence. We show that the fidelity of distillation of a single bit of coherence under SIO can be efficiently computed as a semidefinite program, and investigate the generalization of this result to provide an understanding of asymptotically achievable distillation fidelity.
\end{abstract}

DOI: 10.1103/PhysRevLett.122.150402

Introduction.-The resource theory of quantum coherence [1-4] has found extensive use in the characterization of a signature intrinsic feature of quantum mechanicssuperposition-and our ability to manipulate it efficiently within a resource-theoretic framework [5-8]. Typically, the properties of a resource are investigated under a suitable set of allowed free operations, reflecting the constraints placed on the manipulation of the given resource [5,8]. In spite of the fact that the resource theory of coherence has found use in a variety of practical settings [4], no physically compelling set of assumptions has yet emerged which could single out a unique class of free operations under which the operational features of coherence should be investigated, mirroring the fundamental role of local operations and classical communication in the resource theory of entanglement [9]. This has motivated the definition and characterization of a multitude of possible sets of free operations, and sparked efforts to compare their operational power [10-26]. However, many definitions of free operations stemming from meaningful physical considerations, such as physically incoherent operations (PIO) [13], translationally covariant incoherent operations [14], or genuinely incoherent operations [16], were found to be too limited in their operational capabilities, suggesting that any useful resource theory of coherence would require a larger set of maps. On the other hand, strictly larger sets of maps such as maximally incoherent operations (MIO) [1], incoherent operations (IO) [2], or dephasingcovariant incoherent operations (DIO) $[13,14]$, while operationally powerful, might be considered as too permissive and lacking a physically implementable form.

The class of strictly incoherent operations (SIO) $[3,12]$ appeared to be a promising candidate for a natural class of operations satisfying desirable resource-theoretic criteria while at the same time being motivated on physical grounds and experimentally implementable, causing it to find widespread use in the resource theory of coherence $[3,12,15,18,27]$. SIO are easy to characterize and are a seemingly powerful choice of free operations, allowing in particular for a "golden unit" of coherence represented by the maximally coherent state $\left|\Psi_{m}\right\rangle$, which can be transformed into any other state using SIO [2]. Although strictly smaller than the sets IO and DIO, the set SIO includes many more transformations than PIO, and its operational capabilities did not appear to be too limited-for instance, SIO have exactly the same power as IO as far as pure-to-pure state transformations are concerned [15,28], as well as in the context of coherence dilution $[3,19]$; they match the power of DIO in probabilistic distillation from pure states [23], and even the largest class of free operations, MIO, cannot perform better than SIO in one-shot distillation from pure states [20], in assisted coherence distillation [25], and in all single-qubit state transformations [15]. On the other hand, there do exist tasks in which the limitations of SIO become apparent-in particular, unlike the larger sets IO, DIO, and MIO, the class SIO has recently been found to exhibit bound coherence [26]; i.e., there are coherent states from which no coherence can be distilled by such operations. While the same phenomenon was known for PIO, it was arguably unexpected for SIO. It is, however, not known how common this property is among all quantum states, nor to what extent it limits the operational power of SIO beyond specific examples-as is known from entanglement theory [9], the mere existence of undistillable states does not inhibit a class of operations from being useful in manipulating a resource. Indeed, a complete description of 
coherence distillation under SIO has been a long-standing open problem in the resource theory of coherence $[3,4,12,26,29]$, and its solution would shed light on concretely achievable possibilities in coherence manipulation.

In this Letter, we solve this problem completely: namely, we analytically compute the maximal rates of coherence distillation under SIO and PIO, showing that they coincide on all states. By introducing an SIO coherence monotone that does not change when multiple copies of a state are considered, we establish a simple criterion to decide whether a given quantum system is asymptotically distillable or not. In the former case, we derive an upper bound on the SIO distillable coherence and show that it can in fact be achieved by an explicitly constructed PIO protocol. This leads us to the surprising conclusion that the distillable coherence is the same under SIO and PIO. Moreover, it also shows that the optimal SIO distillation protocol can be realized by appending incoherent ancillae, applying incoherent unitaries, and making incoherent measurements; thus, it is easily implementable in practice. Our findings establish in particular that bound coherence is a generic phenomenon. Specifically, we show that almost all quantum states are undistillable under SIO, with the only distillable ones being those whose density matrix contains a submatrix proportional to a pure state. This demonstrates fundamental limitations of SIO in the resource theory of coherence. To arrive at the above results, we introduce a plethora of tools of independent interest, including an efficiently computable semidefinite programming (SDP) expression characterizing the maximal achievable fidelity in the distillation of a single bit of coherence, and an entire new family of SIO monotones. Our Letter substantially advances the theoretical and practical study of quantum coherence.

A new SIO monotone.-Let us begin by recalling the basic formalism of the resource theory of quantum coherence. The set $\mathcal{I}$ of free states, known as incoherent states, consists of all density matrices diagonal in a given $d$ dimensional orthonormal basis $\{|i\rangle\}$. We will denote by $\Delta$ the dephasing map, defined by $\Delta(\cdot)=\sum_{i}|i\rangle\langle i|(\cdot)| i\rangle\langle i|$, and by $\left|\Psi_{m}\right\rangle=(1 / \sqrt{m}) \sum_{i=1}^{m}|i\rangle$ the maximally coherent state of dimension $m$. As for the free operations, we will focus on SIO, defined as those channels $\Lambda$ that admit a Kraus decomposition $\Lambda(\cdot)=\sum_{\alpha} K_{\alpha}(\cdot) K_{\alpha}^{\dagger}$ such that $K_{\alpha} \Delta(\rho) K_{\alpha}^{\dagger}=$ $\Delta\left(K_{\alpha} \rho K_{\alpha}^{\dagger}\right)$ for all $\alpha$ and $\rho$. We will also consider the subset of PIO, which are all maps that admit an incoherent dilation (i.e., they can be implemented by appending an incoherent ancilla and performing incoherent unitaries, permutations, and incoherent projections only) [13].

We now introduce a straightforwardly computable quantity that we name maximal coherence of $\rho$, defined by

$$
\eta(\rho):=\max _{i \neq j} \frac{\left|\rho_{i j}\right|}{\sqrt{\rho_{i i} \rho_{j j}}},
$$

where the optimization is over all choices of indices such that $\rho_{i i} \neq 0 \neq \rho_{j j}$, with $\eta(\rho)=0$ if no such choice exists. Alternatively, this quantity can be understood as the largest modulus of an off-diagonal element of the matrix $\Delta(\rho)^{-1 / 2} \rho \Delta(\rho)^{-1 / 2}$.

We first notice that for all states $\rho$, one has $0 \leq \eta(\rho) \leq 1$. This follows from the positivity of the principal minor of $\rho$ of order 2 corresponding to the rows and columns identified by indices $i$ and $j$, which implies that $\rho_{i i} \rho_{j j} \geq\left|\rho_{i j}\right|^{2}$ for any choice of $i, j$. Moreover, we see by definition that $\eta(\rho)=0$ if and only if $\rho$ is incoherent, and $\eta(\rho)=1$ if and only if there are indices $1 \leq i \neq j \leq d$ such that $\Pi_{i j} \rho \Pi_{i j}$ is proportional to a pure state with nonvanishing diagonal, where $\Pi_{i j}$ is the projector onto $\operatorname{span}\{|i\rangle,|j\rangle\}$.

An important property of $\eta$ is that it is, in fact, monotonically nonincreasing under SIO. Precisely, consider a strictly incoherent operation $\Lambda$ acting on a $d$-dimensional system, which can be written as

$$
\Lambda(\cdot)=\sum_{\alpha} U_{\pi_{\alpha}}^{\top} D_{\alpha}(\cdot) D_{\alpha}^{*} U_{\pi_{\alpha}}
$$

where the $\pi_{\alpha}$ are permutations, $U_{\pi_{\alpha}}:=\sum_{i=1}^{d}\left|\pi_{\alpha}(i)\right\rangle\langle i|$ are the unitaries that implement them, and the matrices $D_{\alpha}:=$ $\sum_{i=1}^{d} d_{\alpha}(i)|i\rangle\langle i|$ are all diagonal. This representation has some technical issues when input and output dimensions are different, but this is irrelevant for the present argument [30]. For two arbitrary indices $1 \leq i \neq j \leq d$, we can write

$$
\begin{aligned}
\left|\Lambda(\rho)_{i j}\right| & \stackrel{\text { (i) }}{=}\left|\sum_{\alpha} d_{\alpha}\left(\pi_{\alpha}(i)\right) d_{\alpha}\left(\pi_{\alpha}(j)\right)^{*} \rho_{\pi_{\alpha}(i), \pi_{\alpha}(j)}\right| \\
\leq & \sum_{\alpha}\left|d_{\alpha}\left(\pi_{\alpha}(i)\right)\right|\left|d_{\alpha}\left(\pi_{\alpha}(j)\right)\right|\left|\rho_{\pi_{\alpha}(i), \pi_{\alpha}(j)}\right| \\
\stackrel{(\text { ii })}{\leq} & \eta(\rho) \sum_{\alpha}\left|d_{\alpha}\left(\pi_{\alpha}(i)\right)\right|\left|d_{\alpha}\left(\pi_{\alpha}(j)\right)\right| \\
& \times \sqrt{\rho_{\pi_{\alpha}(i), \pi_{\alpha}(i)}} \sqrt{\rho_{\pi_{\alpha}(j), \pi_{\alpha}(j)}} \\
& \stackrel{(\text { iii) }}{\leq} \eta(\rho)\left\{\sum_{\alpha}\left|d_{\alpha}\left(\pi_{\alpha}(i)\right)\right|^{2} \rho_{\pi_{\alpha}(i), \pi_{\alpha}(i)}\right\}^{1 / 2} \\
& \times\left\{\sum_{\alpha}\left|d_{\alpha}\left(\pi_{\alpha}(j)\right)\right|^{2} \rho_{\pi_{\alpha}(j), \pi_{\alpha}(j)}\right\}^{1 / 2} \\
\stackrel{\text { (iv) }}{=} & \eta(\rho) \sqrt{\Lambda(\rho)_{i i} \Lambda(\rho)_{j j}} .
\end{aligned}
$$

The above steps are justified as follows: (i) we employed the Kraus representation of Eq. (2); (ii) since $i \neq j$ by hypothesis, for all permutations $\pi_{\alpha}$ we have also $\pi_{\alpha}(i) \neq$ $\pi_{\alpha}(j)$ and hence $\left|\rho_{\pi_{\alpha}(i), \pi_{\alpha}(j)}\right| \leq \eta(\rho) \sqrt{\rho_{\pi_{\alpha}(i), \pi_{\alpha}(i)}} \sqrt{\rho_{\pi_{\alpha}(j), \pi_{\alpha}(j)}}$; (iii) we applied the Cauchy-Schwarz inequality; (iv) we resorted once more to the representation in Eq. (2).

Another important property of $\eta$ is its lower semicontinuity (l.s.c.), that is, the fact that for any sequence $\left\{\rho_{k}\right\}_{k}$ 
converging to $\rho$, we have $\eta(\rho) \leq \lim _{\inf _{k \rightarrow \infty}} \eta\left(\rho_{k}\right)$. This follows by noting that $\eta$ can be expressed the maximum over indices $i \neq j$ of the functions $\rho \mapsto f_{i j}(\rho) g_{i j}(\rho)$, where $f_{i j}(\rho)=\left|\rho_{i j}\right|$, while $g_{i j}(\rho)$ is defined as $g_{i j}(\rho)=1 / \sqrt{\rho_{i i} \rho_{j j}}$ if both $\rho_{i i}, \rho_{j j} \neq 0$, and $g_{i j}(\rho)=0$ otherwise. Both $f_{i j}$ and $g_{i j}$ can be noticed to be nonnegative 1.s.c. functions, which means that their product will also be 1.s.c.; $\eta$ is then a maximum of a finite family of 1.s.c. functions, and thus is 1.s.c. itself.

We remark that the measure $\eta$ can be related to similarly defined measures of maximal correlation between classical random variables [31-33] and quantum states [34-37]. In particular, observe that the maximal coherence measure introduced here can in fact be computed as the quantum maximal correlation [34] of the corresponding maximally correlated state $\rho^{\prime}=\sum_{i, j} \rho_{i j}|i i\rangle\langle j j|$. However, importantly, the monotonicity of the former under SIO does not follow from the monotonicity of the latter under local operations, as not all SIO acting on $\rho$ correspond to local operations at the level of $\rho^{\prime}$.

Distillability criterion.-The task of coherence distillation $[3,20,26]$ is concerned with the conversion of general quantum states into maximally coherent states $\Psi_{m}$. The error in the distillation of a state under a set of quantum channels $\mathcal{O}$ is characterized by the fidelity of distillation

$$
F_{\mathcal{O}}(\rho, m):=\sup _{\Lambda \in \mathcal{O}} F\left(\Lambda(\rho), \Psi_{m}\right),
$$

where $F(\sigma, \omega):=\|\sqrt{\sigma} \sqrt{\omega}\|_{1}^{2}$. The (asymptotic) distillable coherence is then the maximal rate at which independent and identically distributed copies of a quantum state can be transformed into copies of the maximally coherent qubit state $\Psi_{2}$ (coherence bit) with asymptotically vanishing error; precisely, we have

$$
C_{d, \mathcal{O}}(\rho):=\sup \left\{r \mid \lim _{n \rightarrow \infty} F_{\mathcal{O}}\left(\rho^{\otimes n}, 2^{r n}\right)=1\right\} .
$$

We will say that a state $\rho$ is distillable under $\mathcal{O}$ if $C_{d, \mathcal{O}}(\rho)>0$.

We now make a crucial observation which lets us immediately relate the maximal coherence $\eta$ to the problem of coherence distillation under SIO. It is the fact that $\eta$ obeys the so-called tensorization property [38], that is, $\eta(\rho \otimes \sigma)=\max \{\eta(\rho), \eta(\sigma)\}, \forall \rho, \sigma$. To prove this identity, observe that, according to Eq. (1), computing $\eta(\rho \otimes \sigma)$ corresponds to maximizing the function $\left|(\rho \otimes \sigma)_{i k, j l}\right| /$ $\sqrt{(\rho \otimes \sigma)_{i k, i k}(\rho \otimes \sigma)_{j l, j l}}$ over all pairs of indices $(i, k) \neq(j, l)$, and that this is equivalent to maximizing $\left(\left|\rho_{i j}\right| / \sqrt{\rho_{i i} \rho_{j j}}\right)\left(\left|\sigma_{k l}\right| / \sqrt{\sigma_{k k} \sigma_{l l}}\right)$ over choices such that $i \neq j$ or $k \neq l$. The latter maximum is achieved on pairs either of the form $(i k, j k)$ with $i \neq j$ or of the form $(i k, i l)$ with $k \neq l$, which corresponds precisely to the larger of $\eta(\rho)$ and $\eta(\sigma)$.

By the tensorization and monotonicity of $\eta$, we readily obtain one of our main results: a necessary and sufficient criterion for the distillability of an arbitrary quantum state under SIO.

Theorem 1: For all states $\rho$, the following are equivalent: (a) $C_{d \text {,SIO }}(\rho)>0$, (b) $C_{d, \text { PIO }}(\rho)>0$, and (c) $\eta(\rho)=1$.

Proof: Noting that $\eta\left(\Psi_{2}\right)=1$ and remembering that $\eta$ is 1.s.c., we see that for SIO distillation to be possible, there needs to exist a sequence of SIO operations $\Lambda_{n}$ such that $\eta\left(\lim _{n \rightarrow \infty} \Lambda_{n}\left(\rho^{\otimes n}\right)\right)=1$. However, it holds that

$\eta\left(\lim _{n \rightarrow \infty} \Lambda_{n}\left(\rho^{\otimes n}\right)\right) \stackrel{(\mathrm{i})}{\leq} \liminf _{n \rightarrow \infty} \eta\left(\Lambda_{n}\left(\rho^{\otimes n}\right)\right) \stackrel{(\mathrm{ii})}{\leq} \lim _{n \rightarrow \infty} \eta\left(\rho^{\otimes n}\right) \stackrel{(\mathrm{iii})}{=} \eta(\rho)$,

where (i) is due to the 1.s.c. of $\eta$, (ii) comes from its monotonicity, and (iii) follows from the tensorization property. This shows in particular that any state with $\eta(\rho)<$ 1 is SIO (hence PIO) undistillable. Conversely, the PIO protocol given in the proof of the forthcoming Theorem 4 (see below) shows that every state with $\eta(\rho)=1$ is PIO distillable.

The above Theorem 1 establishes a complete characterization of distillability under SIO and PIO. In particular, it is not difficult to see that any generic quantum state exhibits bound coherence, and that the condition for distillability of a state $\rho$-i.e., the existence of a submatrix of $\rho$ in the basis $\{|i\rangle\}_{i}$ proportional to a pure state-is an extremely restrictive property, satisfied only by a zeromeasure class of mixed states. We stress that the proof of the Theorem in fact establishes the stronger statement that any state $\rho$ such that $\eta(\rho)<1$ cannot be used to distill even a single coherence bit, no matter how large the number of available copies of $\rho$ is. We will see later that this relation between SIO and PIO extends beyond the distillability criterion.

Fidelity of distillation under SIO. - It follows from ([26], Theorem 10) that the fidelity of distillation of an $\mathrm{m}$ dimensional maximally coherent state $\Psi_{m}$ under SIO for any state $\rho$ is

$$
F_{\mathrm{SIO}}(\rho, m)=\max \left\{\operatorname{Tr} \rho A \mid 0 \leq A \leq \mathbb{1}, \Delta(A)=\frac{\mathbb{1}}{m}, \mathrm{CN}(A) \leq m\right\},
$$

where the coherence number $\mathrm{CN}(A)$ of $A \geq 0$ is defined as the minimal integer $r$ such that $A$ can be written as a positive linear combination of rank-one projectors $\left|x_{i}\right\rangle\left\langle x_{i}\right|$ with $\operatorname{rank}\left[\Delta\left(\left|x_{i}\right\rangle\left\langle x_{i}\right|\right)\right] \leq r$ for all $i[41,42]$. For the case of distilling a coherence bit $\Psi_{2}$, we are able to obtain the following simplified characterization.

Theorem 2: The fidelity of distillation of a single bit of coherence under SIO is given by the SDP 


$$
\begin{aligned}
F_{\mathrm{SIO}}(\rho, 2) & =\max _{\substack{1 \leq X \leq 1 \\
-1(X)=0 \\
X \succeq 0}} \frac{1}{2}(\operatorname{Tr}|\rho| X+1) \\
& =\min _{\substack{D=\Delta(D) \\
N \succeq 0}} \frac{1}{2}\left(\||\rho|+D+N\|_{1}+1\right),
\end{aligned}
$$

where $A \succeq 0$ signifies the entrywise inequality $A_{i j} \geq 0$ for all $i, j$, and $|\rho|$ stands for the entrywise modulus of $\rho$.

Proof: We sketch the main idea of the argument, deferring the details to the Supplemental Material [30]. The expression in Eq. (5) for the distillation fidelity involves the nontrivial constraint $\mathrm{CN}(A) \leq m$ on the coherence number of the variable $A \geq 0$. For $m=2$, this can be cast into an analytically manageable form thanks to ([43], Theorem 1) which states that $\mathrm{CN}(A) \leq 2$ iff $2 \Delta(A)-|A| \geq 0$. By leveraging this criterion and choosing carefully the optimization variables, one arrives at Eq. (6a). Finally, Eq. (6b) is obtained by taking the SDP dual.

The above results can be compared with analogous expressions for $F_{\mathrm{MIO}}(\rho, 2)$ and $F_{\mathrm{DIO}}(\rho, 2)$ [20]. In particular, it is known that $F_{\mathrm{MIO}}(\psi, m)=F_{\mathrm{SIO}}(\psi, m)$ for all pure states $\psi$ and all $m$ [20]. It is left to determine how closely one can approximate distillation of a perfect bit of coherence by means of SIO when one is given a large number of copies of an input state. This leads us to investigate the quantity $F_{\text {SIO }}\left(\rho^{\otimes n}, 2\right)$ as a function of $\rho$ and $n$, and in particular its asymptotic properties in the limit of large $n$. The following result, whose full proof we provide in the Supplemental Material [30], provides an operational interpretation of the SIO monotone $\eta$ introduced here.

Theorem 3: For all states $\rho$ it holds that

$$
\lim _{n \rightarrow \infty} F_{\text {SIO }}\left(\rho^{\otimes n}, 2\right)=\frac{1+\eta(\rho)}{2},
$$

and the convergence in the above identity is exponentially fast.

As a particularly strong example of SIO and PIO undistillability, consider the class of qubit states $\rho_{\lambda}=$ $\lambda \Psi_{2}+(1-\lambda)(\mathbb{1} / 2)$ with $\lambda \in[0,1]$. An explicit computation yields $\eta\left(\rho_{\lambda}\right)=\lambda$. By constructing a suitable choice of feasible solutions for the SDP [[30], Eq. (6)], it can be shown that $F_{\mathrm{SIO}}\left(\rho_{\lambda}^{\otimes n}, 2\right)=(1+\lambda) / 2$ for any number of copies $n$. Therefore, not only is the distillation of $\rho_{\lambda}$ impossible under SIO for $\lambda \neq 1$, it actually is impossible to increase the fidelity of distillation whatsoever by adding more copies of the state.

Distillable coherence under SIO and PIO.-Although we have proven that most states are bound coherent under SIO or PIO, it could be nevertheless interesting to compute the amount of coherence $C_{d, \mathrm{SIO}}, C_{d, \mathrm{PIO}}$ that can be extracted from distillable states. This is a very different scenario from that considered in Theorem 3: while there we were interested in the distillation of a single coherence bit with good fidelity, here we look at the maximal rate of distillation of bits of coherence with vanishing errors.

Motivated by the properties of the monotone $\eta(\rho)$, we will now consider a quantifier that we will relate to the distillable coherence. For a state $\rho$ such that $\Delta(\rho)>0$, construct the set $E_{\rho}:=\left\{(i, j):\left|\rho_{i j}\right|=\sqrt{\rho_{i i} \rho_{j j}}\right\}$. As we show in the Supplemental Material [30], it turns out that there is a partition $\left\{I_{s}^{\rho}\right\}_{s \in \mathcal{S}^{\rho}}$ of $\{1, \ldots, d\}$ such that $(i, j) \in$ $E_{\rho}$ if and only if $i, j$ belong to the same set $I_{s}^{\rho}$. With this observation, one can show that the operator $\bar{\rho}:=$ $\sum_{(i, j) \in E_{\rho}} \rho_{i j}|i\rangle\langle j|$ is a legitimate density matrix, and that the quantifier

$$
Q(\rho):=S(\Delta(\rho))-S(\bar{\rho})
$$

is (i) non-negative, (ii) strictly positive iff $\eta(\rho)=1$, and (iii) additive over tensor products.

We will now show that $Q$ in fact exactly quantifies the SIO and PIO distillable coherence of any state. The result will strengthen the relation between these two classes of operations, showing that-in contrast to task such as coherence dilution, where SIO are as powerful as larger sets of operations, in distillation the power of SIO is actually the same as PIO, where the latter are known to define a very limited framework [15]. We note that coherence distillation under PIO beyond pure states has not been characterized before in any way [4]. As usual, the process of evaluating a maximal distillation rate is composed of two parts. First, one designs a protocol that achieves the conjectured rate in the limit of a large number of copies (direct part). Second, one shows that the performance of this protocol can not be beaten at least asymptotically (converse part).

Theorem 4: For all states $\rho$, the distillable coherence under SIO and PIO satisfies $C_{d, \mathrm{SIO}}(\rho)=C_{d, \mathrm{PIO}}(\rho)=Q(\rho)$.

Proof: To establish that $Q(\rho)$ gives a lower bound to the rate of PIO distillation, given $n$ copies of the state $\rho$, we perform independently on each of them the measurement $\left\{\Pi_{I_{s}^{\rho}}\right\}_{s \in \mathcal{S}^{\rho}}$, where $\Pi_{I_{s}^{p}}:=\sum_{i \in I_{s}^{\rho}}|i\rangle\langle i|$ and $\left\{I_{s}^{\rho}\right\}_{s \in \mathcal{S}^{\rho}}$ is the partition of $\{1, \ldots, d\}$ identified above. Setting $P(s):=$ $\operatorname{Tr}\left[\rho \Pi_{I_{s}^{\rho}}\right]$ and $\bar{\rho}_{s}:=P(s)^{-1} \Pi_{I_{s}^{\rho}} \rho \Pi_{I_{s}^{\rho}}$, we see that this protocol produces an average of $n P(s)$ copies of the states $\bar{\rho}_{s}$, which can be shown to be all pure. It is known [15] that there exists a PIO protocol that extracts $S(\Delta(\psi))$ coherence bits per copy out of any pure state $\psi$. Applying this procedure to each $\bar{\rho}_{s}$ leads to an expected number of coherence bits produced equal to $\sum_{s \in \mathcal{S}^{\circ}} n P(s) S\left(\Delta\left(\bar{\rho}_{s}\right)\right)=n Q(\rho)$, achieving a rate $Q(\rho)$. See the Supplemental Material [30] for further technical details.

To show the converse, consider the family $\left\{I_{s}^{\rho}\right\}_{s \in \mathcal{S}^{\rho}}$ of disjoint subsets of $[d]$ as discussed above. For any other 
state $\sigma$, we can then construct a random variable $S_{\sigma}^{\rho}$ supported on $\mathcal{S}^{\rho}$ whose probability distribution takes the form $P_{S_{\sigma}^{\rho}}(s):=\operatorname{Tr}\left[\sigma \Pi_{I_{s}^{p}}\right]$. Clearly, $S_{\sigma}^{\rho}$ is a coarse-grained version of the random variable $I_{\sigma}$ distributed according to $P_{I_{\sigma}}(i):=\sigma_{i i}=\langle i|\sigma| i\rangle$. A first important observation is that the quantifier $Q$ coincides with the conditional entropy of $I_{\rho}$ given $S_{\rho}^{\rho}: Q(\rho)=H\left(I_{\rho} \mid S_{\rho}^{\rho}\right)$. To establish that $Q$ gives the asymptotic rate of distillation exactly, we will employ the family of monotones defined as [44]

$$
\mu_{k}(\rho):=\max _{I \subseteq[d],|I| \leq k} \log \left\|\Pi_{I} \Delta(\rho)^{-1 / 2} \rho \Delta(\rho)^{-1 / 2} \Pi_{I}\right\|_{\infty},
$$

where for $I \subseteq[d]$ we set $\Pi_{I}:=\sum_{i \in I}|i\rangle\langle i|$, and the inverse of $\Delta(\rho)$ is taken on the support. These functions can be thought of as a generalization of the previously introduced $\eta$, as $\mu_{2}(\rho)=\log [1+\eta(\rho)]$. The proof proceeds by showing that by suitably smoothing the quantities $\mu_{k}$, they can be related with a family of smoothed conditional max entropies, which then can be related to $H\left(I_{\rho} \mid S_{\rho}^{\rho}\right)$ by establishing a tweaked asymptotic equipartition property. Using the monotonicity of the family $\mu_{k}$ under SIO, we can then show that in the limit of infinitely many copies of $\rho$ the achievable rates of distillation under SIO are constrained precisely as $C_{d \text {,SIO }}(\rho) \leq Q(\rho)$. We refer to Refs. [30,44] for the complete technical details of the proof.

Conclusions.-We fully characterized the problem of asymptotic distillability of quantum coherence under SIO and PIO, analytically computing the maximal asymptotic distillation rates and showing that they coincide on all states. We showed that almost all states-with the sole exception of states whose density matrix contains a rankone submatrix - are bound coherent. A new SIO monotone, the maximal coherence $\eta$, plays a crucial role in forming a necessary and sufficient criterion for distillability. We furthermore derived a computable SDP expression for the fidelity of one-shot distillation of a coherence bit under SIO and evaluated it in the asymptotic many-copy limit in terms of the monotone $\eta$.

Our results reveal that, despite being as useful as the larger classes of free operations IO, DIO, and MIO in some tasks, the operational capabilities of SIO and PIO are limited in the context of coherence distillation. This a priori unexpected conclusion was not suggested by any previous work, and bears a notable impact on practical applications, which often require the use of coherence in pure, distilled form [11,45-47]. For those states that happen to be SIO distillable, we constructed a protocol to perform optimal distillation, that should be easily implementable as it requires only incoherent ancillae, incoherent unitaries, and incoherent measurements.

We note the similarity of our main result to Ref. [48], where a generic phenomenon of bound coherence was also found in the related resource theory of unspeakable coherence (a.k.a. asymmetry) with respect to the set of translationally covariant incoherent operations [14]; however, it does not appear possible to make this qualitative correspondence also quantitative, as the two settings are fundamentally different.

In light of the considerations presented in our Letter and the exposed weakness of SIO in performing coherence distillation, it remains an important open question to understand what the smallest physically motivated set of free operations for manipulating coherence without such hindering operational limitations could be, and hence the ongoing quest for a satisfactory resource theory of coherence [4] becomes even more enthralling.

We are grateful to Iman Marvian, Alexander Streltsov, and Andreas Winter for useful discussions, and to the authors of Ref. [26] for sharing a preliminary draft of their work with us. We thank the Isaac Newton Institute for Mathematical Sciences for support and hospitality during the program "Beyond I.I.D. in Information Theory" when part of the work on this Letter was undertaken. We acknowledge financial support from the European Research Council (ERC) under the Starting Grant GQCOP (Grant No. 637352).

*ludovico.lami@gmail.com

†bartosz.regula@gmail.com

†gerardo.adesso@nottingham.ac.uk

[1] J. Aberg, arXiv:quant-ph/0612146.

[2] T. Baumgratz, M. Cramer, and M. B. Plenio, Phys. Rev. Lett. 113, 140401 (2014).

[3] A. Winter and D. Yang, Phys. Rev. Lett. 116, 120404 (2016).

[4] A. Streltsov, G. Adesso, and M. B. Plenio, Rev. Mod. Phys. 89, 041003 (2017).

[5] M. Horodecki and J. Oppenheim, Int. J. Mod. Phys. B 27, 1345019 (2013).

[6] L. del Rio, L. Kraemer, and R. Renner, arXiv:1511.08818.

[7] B. Coecke, T. Fritz, and R. W. Spekkens, Inf. Comput. 250, 59 (2016).

[8] E. Chitambar and G. Gour, arXiv:1806.06107.

[9] R. Horodecki, P. Horodecki, M. Horodecki, and K. Horodecki, Rev. Mod. Phys. 81, 865 (2009).

[10] S. Du, Z. Bai, and X. Qi, Quantum Inf. Comput. 15, 1307 (2015).

[11] X. Yuan, H. Zhou, Z. Cao, and X. Ma, Phys. Rev. A 92, 022124 (2015).

[12] B. Yadin, J. Ma, D. Girolami, M. Gu, and V. Vedral, Phys. Rev. X 6, 041028 (2016).

[13] E. Chitambar and G. Gour, Phys. Rev. Lett. 117, 030401 (2016).

[14] I. Marvian and R. W. Spekkens, Phys. Rev. A 94, 052324 (2016).

[15] E. Chitambar and G. Gour, Phys. Rev. A 94, 052336 (2016).

[16] J. I. de Vicente and A. Streltsov, J. Phys. A 50, 045301 (2017).

[17] Z.-W. Liu, X. Hu, and S. Lloyd, Phys. Rev. Lett. 118, 060502 (2017). 
[18] A. Streltsov, S. Rana, P. Boes, and J. Eisert, Phys. Rev. Lett. 119, 140402 (2017).

[19] Q. Zhao, Y. Liu, X. Yuan, E. Chitambar, and X. Ma, Phys. Rev. Lett. 120, 070403 (2018).

[20] B. Regula, K. Fang, X. Wang, and G. Adesso, Phys. Rev. Lett. 121, 010401 (2018).

[21] E. Chitambar, Phys. Rev. A 97, 050301(R) (2018).

[22] D. Egloff, J. M. Matera, T. Theurer, and M. B. Plenio, Phys. Rev. X 8, 031005 (2018).

[23] K. Fang, X. Wang, L. Lami, B. Regula, and G. Adesso, Phys. Rev. Lett. 121, 070404 (2018).

[24] T. Theurer, D. Egloff, L. Zhang, and M. B. Plenio, arXiv: 1806.07332.

[25] B. Regula, L. Lami, and A. Streltsov, Phys. Rev. A 98, 052329 (2018).

[26] Q. Zhao, Y. Liu, X. Yuan, E. Chitambar, and A. Winter, arXiv:1808.01885v2.

[27] T. Biswas, M. García Díaz, and A. Winter, Proc. R. Soc. A 473, 20170170 (2017).

[28] S. Du, Z. Bai, and Y. Guo, Phys. Rev. A 91, 052120 (2015).

[29] K. Ben Dana, M. García Díaz, M. Mejatty, and A. Winter, Phys. Rev. A 95, 062327 (2017).

[30] See Supplemental Material at http://link.aps.org/ supplemental/10.1103/PhysRevLett.122.150402 for explicit proofs of some of the results discussed in the main text.

[31] H. O. Hirschfeld, Math. Proc. Cambridge Philos. Soc. 31, 520 (1935).

[32] H. Gebelein, Z. Angew. Math. Mech. 21, 364 (1941).
[33] H. Witsenhausen, SIAM J. Appl. Math. 28, 100 (1975).

[34] S. Beigi, J. Math. Phys. (N.Y.) 54, 082202 (2013).

[35] P. Delgosha and S. Beigi, Commun. Math. Phys. 332, 449 (2014).

[36] S. Beigi and A. Gohari, IEEE Trans. Inf. Theory 61, 5185 (2015).

[37] S. Beigi and A. Gohari, IEEE Trans. Inf. Theory 64, 2193 (2018).

[38] We note that the existence of a monotone obeying the tensorization property has also been used to study distillation of quantum resources in continuous variable systems [39,40].

[39] G. Giedke and J. Ignacio Cirac, Phys. Rev. A 66, 032316 (2002).

[40] L. Lami, B. Regula, X. Wang, R. Nichols, A. Winter, and G. Adesso, Phys. Rev. A 98, 022335 (2018).

[41] S. Chin, Phys. Rev. A 96, 042336 (2017).

[42] B. Regula, M. Piani, M. Cianciaruso, T. R. Bromley, A. Streltsov, and G. Adesso, New J. Phys. 20, 033012 (2018).

[43] M. Ringbauer, T. R. Bromley, M. Cianciaruso, L. Lami, W. Y. S. Lau, G. Adesso, A. G. White, A. Fedrizzi, and M. Piani, Phys. Rev. X 8, 041007 (2018).

[44] L. Lami, arXiv:1902.02427.

[45] A. Streltsov, E. Chitambar, S. Rana, M. N. Bera, A. Winter, and M. Lewenstein, Phys. Rev. Lett. 116, 240405 (2016).

[46] A. Anshu, R. Jain, and A. Streltsov, arXiv:1804.04915.

[47] M. G. Daz, K. Fang, X. Wang, M. Rosati, M. Skotiniotis, J. Calsamiglia, and A. Winter, Quantum 2, 100 (2018).

[48] I. Marvian, arXiv:1805.01989. 